\title{
DNA damage is a novel response to sublytic complement C5b-9-induced injury in podocytes
}

\author{
Jeffrey W. Pippin, Raghu Durvasula, Arndt Petermann, Keiju Hiromura, \\ William G. Couser, and Stuart J. Shankland \\ Department of Medicine, Division of Nephrology, University of Washington, Seattle, Washington, USA
}

In response to $\mathrm{Ab}$-complement-mediated injury, podocytes can undergo lysis, apoptosis, or, when exposed to sublytic ( $<5 \%$ lysis) amounts of C5b-9, become activated. Following the insertion of sublytic quantities of C5b-9, there is an increase in signaling pathways and growth factor synthesis and release of proteases, oxidants, and other molecules. Despite an increase in DNA synthesis, however, sublytic C5b-9 is associated with a delay in $\mathrm{G}_{2} / \mathrm{M}$ phase progression in podocytes. Here we induced sublytic C5b-9 injury in vitro by exposing cultured rat podocytes or differentiated postmitotic mouse podocytes to $\mathrm{Ab}$ and a complement source; we also studied the passive Heymann nephritis model of experimental membranous nephropathy in rats. A major finding was that sublytic C5b-9-induced injury caused an increase in DNA damage in podocytes both in vitro and in vivo. This was associated with an increase in protein levels for $\mathrm{p} 53$, the CDK inhibitor $\mathrm{p} 21$, growth-arrest DNA damage-45 (GADD45), and the checkpoint kinases-1 and -2. Sublytic C5b-9 increased extracellular signal-regulated kinase- 1 and -2 (ERK-1 and -2), and inhibiting ERK-1 and -2 reduced the increase in p21 and GADD45 and augmented the DNA damage response to sublytic C5b-9-induced injury. These results show that sublytic C5b-9 induces DNA damage in vitro and in vivo and may explain why podocyte proliferation is limited following immune-mediated injury.

J. Clin. Invest. 111:877-885 (2003). doi:10.1172/JCI200315645.

\section{Introduction}

The visceral glomerular epithelial cell, or podocyte, is a terminally differentiated cell on the outer aspect of the glomerular basement membrane. Podocytes are the target of immune-mediated injury and nonimmune injury, such as toxins, viruses, stress tension, and permeability factors. The complement system is a potent mediator of glomerular injury in immune complex glomerulonephritis. The number of C5b-9 (membrane attack) complexes inserted into cell membranes determines whether cells undergo lysis (also called necrosis) (1). Consequences of sublytic C5b-9 attack include the release of calcium, activation of specific signaling pathways (2), an increase in specific growth factors, matrix components (3), oxidants and proteases $(4,5)$, and under certain circumstances, apoptosis. Podocytes enter the cell cycle following sublytic injury

Received for publication April 9, 2002, and accepted in revised form January 14, 2003.

Address correspondence to: Stuart J. Shankland, Division of Nephrology, University of Washington, Box 356521, Seattle, Washington 98195, USA. Phone: (206) 543-3792;

Fax: (206) 685-8661; E-mail: stuartjs@u.washington.edu.

Conflict of interest: The authors have declared that no conflict of interest exists.

Nonstandard abbreviations used: passive Heymann nephritis (PHN); checkpoint kinase (CHK); growth-arrest DNA damage-45 (GADD45); extracellular signal-regulated kinase (ERK); rat mesangial cell (RMC); Piebald Virol Glaxo (PVG); functional complement system present $\left(\mathrm{C}^{+}\right)$; functional complement system absent $\left(\mathrm{C}^{-}\right)$; lactate dehydrogenase (LDH); passive Heymann nephritis (PHN); apurinic-apyrimidinic (AP); single-stranded DNA (ssDNA); aldehyde reactive probe reagent (ARP); cyclindependent kinase (CDK). and undergo DNA synthesis, albeit limited (6). In contrast to mesangial and certain nonrenal cells, however, podocytes do not typically undergo mitosis following sublytic C5b-9 injury $(7,8)$.

The mechanisms underlying the apparent inability of podocytes to proliferate following sublytic C5b-9induced injury have not been well elucidated. Based on the characteristic finding of podocyte polyploidy present in diseases of the podocyte, such as experimental membranous nephropathy (9), some have suggested that there is a defect in mitosis. Recent studies have shown that DNA damage induced by UV irradiation is associated with cell cycle arrest, which permits cells sufficient time for appropriate DNA repair mechanisms to occur (10). Inadequate repair results in the propagation of damaged DNA to daughter cells, with undesirable consequences.

Here we present data that a novel consequence of sublytic C5b-9 injury to podocytes is DNA damage. Our results show that sublytic C5b-9 induces DNA damage in mouse and rat podocytes in vitro, as well as in vivo in the passive Heymann nephritis (PHN) model of membranous nephropathy. We also show that specific cell cycle-related genes, including p53, p21, growth-arrest DNA damage-45 (GADD45), checkpoint kinase-1 (CHK-1) and CHK-2, are activated by sublytic C5b-9. In addition we show that the extracellular signal-regulated kinase (ERK) is a critical pathway involved in regulating these cell cycle-related proteins following C5b-9-induced DNA damage in podocytes and that ERK protects podocytes from DNA damage. 


\section{Methods}

\section{Cells in culture}

Rat podocytes. Rat visceral glomerular epithelial cells, also called podocytes, were established in culture as described previously (6). In certain experiments, quiescent rat podocytes were studied to more closely resemble the quiescent in vivo podocyte phenotype by depriving them of growth factors for $48 \mathrm{~h}$. Quiescence was confirmed by FACS analysis and BrdU staining $(11,12)$. Podocytes were exposed to $\mathrm{Ab}$ and a complement source (described below), and DNA damage was measured (described below).

Differentiated postmitotic mouse podocytes. To ensure that the results obtained in rat podocytes also occurred in another species and in cells that better represent cells in vivo, we used well-characterized differentiated postmitotic mouse podocytes (obtained from Peter Mundel, Albert Einstein College of Medicine, New York, New York, USA), referred to as conditionally immortalized mouse podocytes. The methods for producing $\mathrm{H}-2 \mathrm{~K}^{\mathrm{b}}$ tsA58 transgenic mice and isolating podocytes from $\mathrm{H}-2 \mathrm{~K}^{\mathrm{b}}$-tsA58 kidneys have been described previously (13). When these mouse podocytes are cultured under growth-permissive conditions $\left(33^{\circ} \mathrm{C}\right.$ and media supplemented with $50 \mu \mathrm{g} / \mathrm{ml}$ mouse IFN- $\gamma$ ), they proliferate and maintain a uniform, cobblestone morphology. In contrast, when grown under differentiating conditions $\left(37^{\circ} \mathrm{C}\right.$, no IFN- $\left.\gamma\right)$ they stop proliferating, elaborate a complex network of processes, and take on a typically differentiated and postmitotic phenotype. We used the latter conditions for the studies presented here.

Mesangial cells. To determine if the effects of sublytic C5b-9 exposure on DNA damage were specific to podocytes, we also studied rat mesangial cells (RMCs) as described previously (11).

\section{Ab-C5b-9 stimulation of cells in vitro}

Podocytes. To induce sublytic C5b-9 attack, mouse and rat podocytes were plated and allowed to grow to $50 \%$ confluence. Podocytes were divided into three groups (6). Group 1 was sensitized with media containing either $5 \mathrm{mg} / \mathrm{ml}$ of sheep anti-Fx1A IgG (rat podocytes) or $2 \mathrm{mg} / \mathrm{ml}$ sheep anti-mouse podocyte IgG (mouse podocytes) at $37^{\circ} \mathrm{C}$ for $30 \mathrm{~min}$. Sensitized podocytes were then exposed to a sublytic amount of complement (see below) for $60 \mathrm{~min}$, washed three times, and then incubated in growth media. Group 2, the control for C5b-9-attacked cells, were sensitized with $\operatorname{IgG}$ and exposed to serum that lacked C6 for $60 \mathrm{~min}$ (see below). Group 3 podocytes, the control for the Ab's, were exposed to media at $37^{\circ} \mathrm{C}$ for $30 \mathrm{~min}$ in the absence of either anti-Fx1A or anti-mouse podocytes.

RMCs. To determine if sublytic C5b-9 attack also induced DNA damage in other cell types, RMCs plated in a 24 -well plate at a density of $2 \times 10^{5}$ cells $/ \mathrm{cm}^{2}$ were incubated with anti-Thy $1 \mathrm{IgG}(5 \mathrm{mg} / \mathrm{ml})$ at $37^{\circ} \mathrm{C}$ for 30 $\min (11)$. One group was then exposed to a sublytic amount of complement (PVG $\mathrm{C}^{+}$serum, see below), while control cells were exposed to serum lacking C6 (PVG C ${ }^{-}$serum, see below).

Reagents and conditions for sublytic C5b-9 attack

Serum from normal Piebald Virol Glaxo (PVG) rats (Harlan Sprague-Dawley, Indianapolis, Indiana, USA) with a functional complement system present $\left(\mathrm{C}^{+}\right)$was used as a complement source to induce sublytic C5b-9 attack. Serum from C6-deficient PVG rats with a functional complement system absent $\left(\mathrm{C}^{-}\right)$(Bantin and Kingman Universal, Fremont, California, USA) was used as control. $\mathrm{C}^{-}$rats exhibit complete absence of $\mathrm{C6}$, inherited in an autosomal recessive pattern, and are unable to assemble C5b-9 (14). The selection of the Ab and complement concentrations was based on checkerboard titration studies to define the amounts of $\mathrm{Ab}$ and complement required without producing podocyte injury or lysis (defined as $>5 \%$ lactate dehydrogenase release; see below). Serum was diluted to $8 \%$ and applied to cells sensitized with $\mathrm{Ab}$ and incubated for $1 \mathrm{~h}$ at $37^{\circ} \mathrm{C}$ to allow C5b-9 insertion. Cells were washed with serum-free media to remove unbound complement. To ensure that C5b-9 attack was not inducing significant cell lysis, lactate dehydrogenase (LDH) was measured in the supernatants using the in vitro toxicology assay kit (Sigma-Aldrich, St. Louis, Missouri, USA) (15). Lysis was expressed as a percentage of the change in the absorbance of the media compared with changes in absorbance of the triton-lysed cells and the media $(<5 \%$ LDH release was regarded as sublytic).

\section{Passive Heymann nephritis model}

To test the hypothesis that C5b-9 induced podocyte DNA damage in vivo, the passive Heymann nephritis (PHN) model of experimental membranous nephropathy was induced in male Sprague-Dawley rats (Simonsen Laboratories, Gilroy, California, USA) by intraperitoneal injection $(5 \mathrm{ml} / \mathrm{kg}$ body weight) of sheep $\mathrm{Ab}$ to Fx1A prepared as described previously. Sprague-Dawley rats injected with normal sheep serum $(5 \mathrm{ml} / \mathrm{kg}$ body weight) served as controls for the anti-Fx1A Ab. To confirm that our results were indeed C5b-9 dependent, $\mathrm{PHN}$ was also induced in PVG $\mathrm{C}^{+}$rats and in PVG $\mathrm{C}^{-}$rats. Control and $\mathrm{PHN}$ rats were sacrificed at 3, 5, and 30 days ( $n=6$ at each time point) for renal biopsies and glomerular isolation. Urine protein excretion prior to sacrifice was determined in control and PHN animals using the sulphosalicylic acid method (16).

\section{Measuring DNA damage in vitro and in vivo}

In vitro. Three methods were used to determine the presence of DNA damage in complement-stimulated podocytes in vitro. A DNA precipitation assay (17), single-cell gel electrophoresis assay, and apurinic-apyrimidinic (AP) site counting assay were used.

For the DNA precipitation assay, podocytes were plated into 24 -well plates with $2 \mu \mathrm{Ci}$ of ${ }^{3} \mathrm{H}$-thymidine for every 1 $\mathrm{ml}$ of media. Podocytes were allowed to grow for 3-4 days to incorporate ${ }^{3} \mathrm{H}$-thymidine into the DNA. Following stimulation with $\mathrm{Ab}$ and $\mathrm{C}^{+}$or $\mathrm{C}^{-}$serum, podocytes were 
incubated in media for $30 \mathrm{~min}$ to $24 \mathrm{~h}$, then dissolved using a lysis buffer. $\mathrm{KCl}(12 \mathrm{M})$ was added to lysed cells and incubated at $65^{\circ} \mathrm{C}$ for $10 \mathrm{~min}$, iced for $5 \mathrm{~min}$, then centrifuged at $3,200 \mathrm{~g}$ to separate precipitated chromatin DNA from damaged DNA (supernatant). Supernatants and DNA pellets were dissolved in scintillation fluid and counted in a Beckman 3500 scintillation counter. The percentage of single-stranded DNA (ssDNA) was calculated by dividing the counts per minute in the supernatant by the counts per minute in the supernatant plus the counts per minute in the pellet, and multiplying by 100 .

The single-cell gel electrophoresis assay, or the comet assay (Trevigen, Gaithersburg, Maryland, USA) was also used. Podocytes were scraped $2 \mathrm{~h}$ after complement stimulation, diluted in low-melting point agarose, and applied to a microscope slide provided in the comet assay kit. Slides were incubated in lysis solution followed by an alkali solution to uncoil DNA. Slides were placed in a horizontal gel electrophoresis box, and $27 \mathrm{~V}$ was applied for $10 \mathrm{~min}$. The ssDNA migrated away from the nucleus and were visualized using a fluorescent DNA stain. Images of the nuclei with the characteristic "tail" of damaged DNA were captured under fluorescent microscopy.

AP site counting was performed using the DNA damage-quantification kit from Dojindo Molecular Technologies Inc. (Gaithersburg, Maryland, USA). DNA was isolated from C5b-9-stimulated and control podocytes using the DNeasy isolation kit (QIAGEN Inc., Valencia, California, USA). AP sites were detected in isolated DNA using an aldehyde-reactive probe reagent (ARP). After treating the DNA with ARP reagent, AP sites became tagged with biotin, and the DNA was bound to a microtiter plate along with ARP-tagged DNA standards. The sites were visualized using peroxidase streptavidin. The number of AP sites in the sample DNA were determined by comparing absorbance at $650 \mathrm{~nm}$ of sample DNA to the standard curve.

In vivo. The comet assay (see above) was performed on isolated glomeruli to determine if DNA damage was occurring in podocytes in vivo.

\section{TUNEL assay and DNA fragmentation}

The use of the TUNEL assay has been reported previously (18). The suicide track DNA ladder kit (Oncogene Research Products, Boston, Massachusetts, USA) was used to determine if characteristic DNA fragmentation of apoptosis occurred in complement-stimulated podocytes in vitro. UV irradiation $(5,000 \mathrm{~mJ})$ was used as a positive control. DNA was extracted and apoptotic DNA was separated from high-molecular-weight chromatin using the extraction buffer. The fragmented DNA was precipitated using Pellet Paint coprecipitant, according to the manufacturer's protocol. DNA was then exposed to electrophoresis in an agarose gel containing ethidium bromide $(0.5 \mu \mathrm{g} / \mathrm{ml})$ at $50 \mathrm{~V}$.

\section{Immunostaining}

Renal biopsies were fixed in either methyl Carnoy's solution or formalin and embedded in paraffin. To ensure equal $\mathrm{Ab}$ binding in control and PHN rats, immunofluorescent staining was performed and quantitated for sheep IgG and C5b-9 (19). Indirect immunoperoxidase staining (20) was performed on $4-\mu \mathrm{m}$ sections using the following primary Ab's for overnight incubation: clone PAb421 mouse monoclonal to murine p53, sheep polyclonal anti-CHK-1, rabbit polyclonal anti-CHK-2 (Oncogene Research Products), rabbit polyclonal anti-GADD45 (Santa Cruz Biotechnology Inc., Santa Cruz, California, USA), clone SX118 mouse monoclonal to p21 (Pharmingen, San Diego, California, USA). Controls included omitting the primary $\mathrm{Ab}$ or substituting the primary Ab with preimmune serum. p53 immunostaining was performed on frozen sections by indirect immunofluorescent staining.

\section{Western blot analysis}

Western blot analysis was performed as described previously $(12,19,21)$ on protein extracted from cultured mouse and rat podocytes exposed to sublytic C5b-9 attack and from isolated glomeruli from control and PHN rats. Protein extracts $(20 \mu \mathrm{g})$ were separated under reduced conditions on a $15 \%, 12 \%$, or $8 \%$ SDS-PAGE gel. Membranes were incubated with Ab's to p21, p53, CHK-1, CHK-2, GADD45, and ERK-1 and -2 (Cell Signalling Technology, Beverly, Massachusetts, USA). Controls included using protein known to be positive or negative for the specific protein being stained or omitting the primary $\mathrm{Ab}$. To ensure equal protein loading, Ab's to the housekeeping proteins tubulin (RBI, Natick, Massachusetts, USA) or actin (Chemicon International, Temecula, California, USA) were used, and wherever phosphorylated proteins were measured, total protein for that product was also measured.

\section{Inhibiting ERK-1 and -2}

Because our results showed that ERK-1 and -2 was phosphorylated by sublytic C5b-9 injury (see below), in select experiments the activity of ERK-1 and -2 was selectively inhibited using U0126 (50 $\mu \mathrm{M}$; Cell Signalling Technology). Finally, to determine the impact of inhibiting ERK-1 and -2 on DNA damage, DNA damage was measured (as described above) in cells exposed to U0126, following the induction of sublytic C5b-9.

\section{Results}

\section{Exposing podocytes to $\mathrm{Ab}$ and complement} induces sublytic attack

There was an increase in $\mathrm{LDH}$ of $4 \%$ in both mouse and rat podocytes exposed to $\mathrm{Ab}$ and a complement source ( $\mathrm{C}^{+}$serum) compared with control cells exposed to $\mathrm{Ab}$ and PVG-C6-deficient ( $\left.\mathrm{C}^{-}\right)$serum (data not shown). Similarly, there was an increase of $4 \% \mathrm{LDH}$ release in RMCs exposed to anti-Thy $1 \mathrm{Ab}$ and $\mathrm{C}^{+}$serum compared with control cells exposed to $\mathrm{Ab}$ and $\mathrm{C}^{-}$serum (data not shown). Thus, we induced sublysis $(<5 \%)$ in mouse and rat podocytes and in RMCs. 

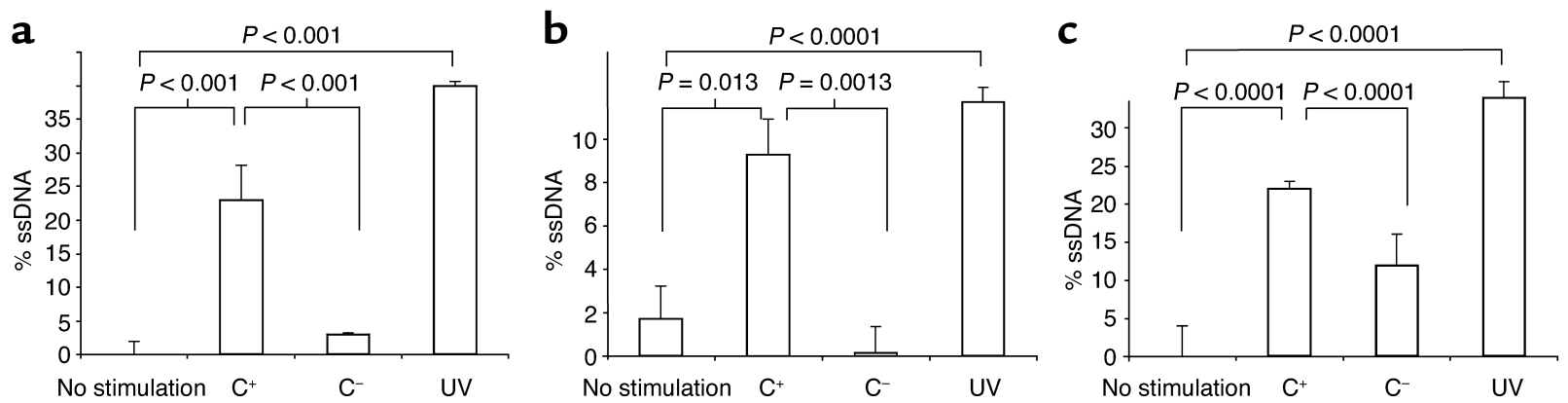

Figure 1

Sublytic C5b-9 causes DNA damage in podocytes in vitro. ssDNA damage was measured by the DNA-precipitation assay in proliferating rat (a), quiescent rat (b), and differentiated postmitotic mouse (c) podocytes. DNA damage was barely detected in control podocytes not exposed to $\mathrm{Ab}$ (lane 1$)$ and in control cells exposed to $\mathrm{Ab}$ without a complement source $\left(\mathrm{C}^{-}\right.$, lane 3$)$. In contrast, DNA damage was significantly increased in podocytes following sublytic C 5 b-9 injury when exposed to $A b$ and a complement source ( $C^{+}$, lane 2$)$. DNA damage was detected in positive control cells exposed to UV irradiation (UV, lane 4). These results show that sublytic C5b-9 injury causes DNA damage in cultured rat and mouse podocytes.

\section{Sublytic C5b-9 injury causes DNA damage in podocytes, but not mesangial cells, in vitro}

DNA damage was measured by three methods. The results of the ssDNA damage assay is shown in Figure 1. Figure 1a shows that exposing proliferating rat podocytes to $\mathrm{Ab}$ and control $\mathrm{C}^{-}$serum was not associated with ssDNA damage. In contrast, there was a significant increase in ssDNA remaining after precipitation in proliferating rat podocytes exposed to $\mathrm{Ab}$ and $\mathrm{a}$ complement source $\left(\mathrm{C}^{+}\right.$serum $)(P<0.001)$. Figure $1 \mathrm{~b}$ shows the DNA damage results in quiescent rat podocytes. Podocytes deprived of serum exited the cell cycle, measured by FACS analysis and BrdU staining (results not shown), similar to podocytes in vivo. Exposing quiescent podocytes to $\mathrm{Ab}$ and $\mathrm{C}^{+}$serum caused a marked increase in DNA damage that was not detected in control quiescent podocytes exposed to $\mathrm{Ab}$ and control $C^{-}$serum $(P<0.001)$. These data show that sublytic C5b-9 causes DNA damage in both proliferating and quiescent cultured rat podocytes. Figure $1 \mathrm{c}$ shows the results obtained in differentiated mouse podocytes. There was a significant increase in ssDNA damage in differentiated postmitotic mouse podocytes exposed to sublytic C5b-9 attack compared with controls.

Figure 2 shows results obtained using the comet assay. DNA damage was not detected in normal rat podocytes (Figure 2a) and control cells exposed to $\mathrm{Ab}$ and $\mathrm{C}^{-}$
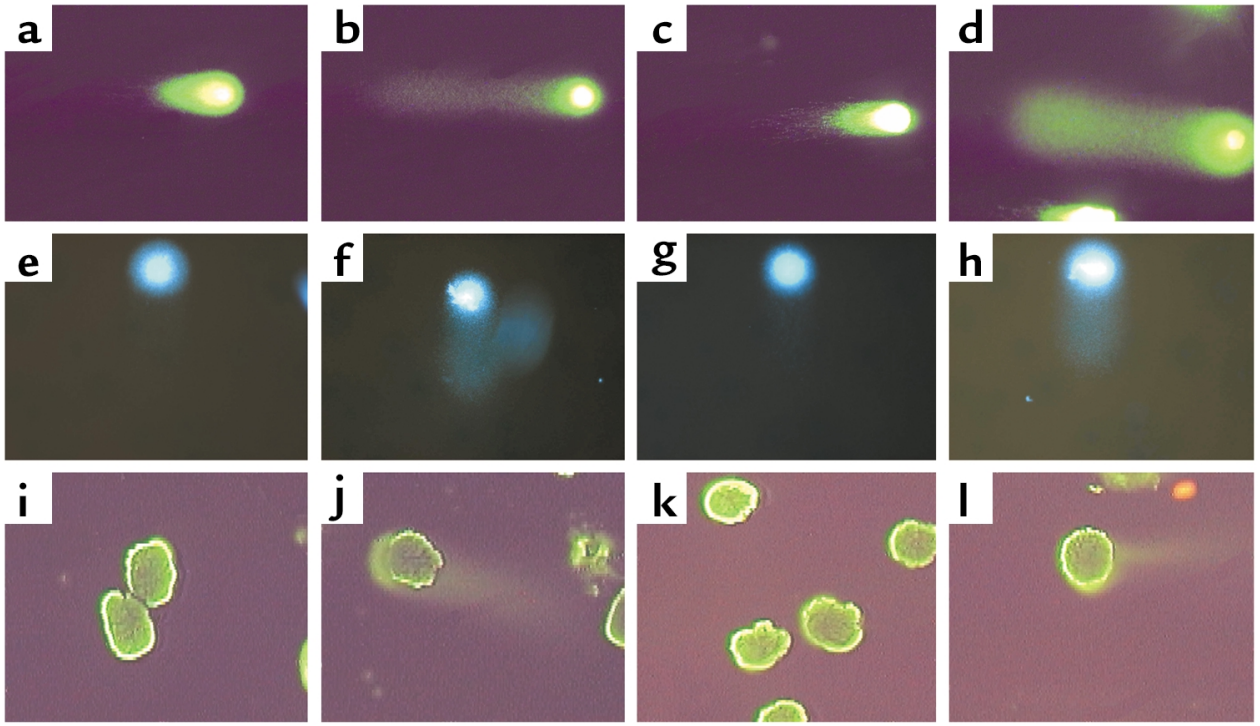

Figure 2

Comet assay. DNA damage was measured in rat (a-d) and mouse (e-h) podocytes in vitro using the comet assay. (a and e) DNA damage was not detected in normal podocytes not exposed to Ab or a complement source. (b and $\mathbf{f}$ ) In podocytes exposed to Ab and complement, the characteristic "tail" of damaged DNA is seen migrating away from the nucleus. (c and $\mathbf{g}$ ) In contrast, DNA damage was not detected in control cells exposed to Ab without a complement source. ( $\mathbf{d}$ and $\mathbf{h}$ ) In UV-irradiated cells, used as a positive control, DNA damage was detected. The comet assay was also performed on glomeruli isolated from control and PHN rats. (i-I) DNA damage was not detected in control rats injected with normal sheep serum (i) nor in PVG $\mathrm{C}^{-}$rats injected with anti-Fx1A Ab (k). DNA damage was detected in Sprague-Dawley PHN (j) and in PVG $C^{+}(\mathbf{I})$ rats injected with anti-Fx1A Ab. 
serum (Figure 2c). The comet assay was positive, however ("tail" of damaged DNA migrating away from the nucleus), in rat podocytes exposed to sublytic C5b-9 (Figure 2b) and in rat podocytes exposed to UV irradiation (Figure 2d) (positive control). The comet assay was negative in normal differentiated mouse podocytes (Figure 2e) and control mouse podocytes exposed to $\mathrm{Ab}$ and $\mathrm{C}^{-}$serum (Figure $2 \mathrm{~g}$ ). In contrast, exposing mouse podocytes to $\mathrm{Ab}$ and a complement source results in a tail (Figure 2f). Mouse podocytes exposed to UV irradiation also exhibited a tail (Figure $2 \mathrm{~h}$ ).

The number of AP sites in DNA from rat podocytes following sublytic C5b-9 exposure was also measured. Podocytes exposed to $\mathrm{Ab}$ and $\mathrm{C}^{+}$serum contained twice the number of AP sites compared with podocytes exposed to $\mathrm{Ab}$ and $\mathrm{C}^{-}$serum ( $40 \pm 1.3$ versus $22 \pm 4.8$; $P<0.001)$. Similar results were obtained using mouse podocytes. These results show that sublytic C5b-9 exposure causes DNA damage in mouse and rat podocytes in vitro.

We also measured DNA damage in RMCs following sublytic C5b-9 exposure. In contrast to podocytes, DNA damage was not detected in RMCs (data not shown). However, UV irradiation, used as a positive control, did induce DNA damage in RMCs (data not shown). Taken together, these results show that sublytic C5b-9 exposure induced DNA damage specifically in podocytes, but not in RMCs.

\section{Sublytic C5b-9 does not induce apoptosis in podocytes}

The results of the TUNEL and suicide track DNA ladder assays used to measure apoptosis are shown for rat (Figure 3, a-c) and mouse (Figure 3, d-f) podocytes. Exposing cultured rat and mouse podocytes to $\mathrm{Ab}$ without a complement source did not induce apoptosis (Figure 3 , a and d). In addition, Figures $3, b$ and e, show that exposing podocytes to $\mathrm{Ab}$ and a complement source was not associated with positive TUNEL staining. The results were not false negatives, because TUNEL staining increased in podocytes exposed to apoptosis-inducing doses of UV irradiation (Figure $3, \mathrm{c}$ and $\mathrm{f}$ ).

The results of DNA laddering measured by the suicide track DNA ladder assay are shown in Figure 3. DNA laddering was not detected in rat (Figure 3g) or mouse (Figure $3 \mathrm{~h}$ ) podocytes exposed to $\mathrm{Ab}$ with or without a complement source. DNA laddering, however, was detected in podocytes exposed to UV irradiation, which was used as a positive control. Taken together, these assays show that sublytic C5b-9-induced injury to rat and mouse podocytes does not cause apoptosis, but rather specifically causes DNA damage.

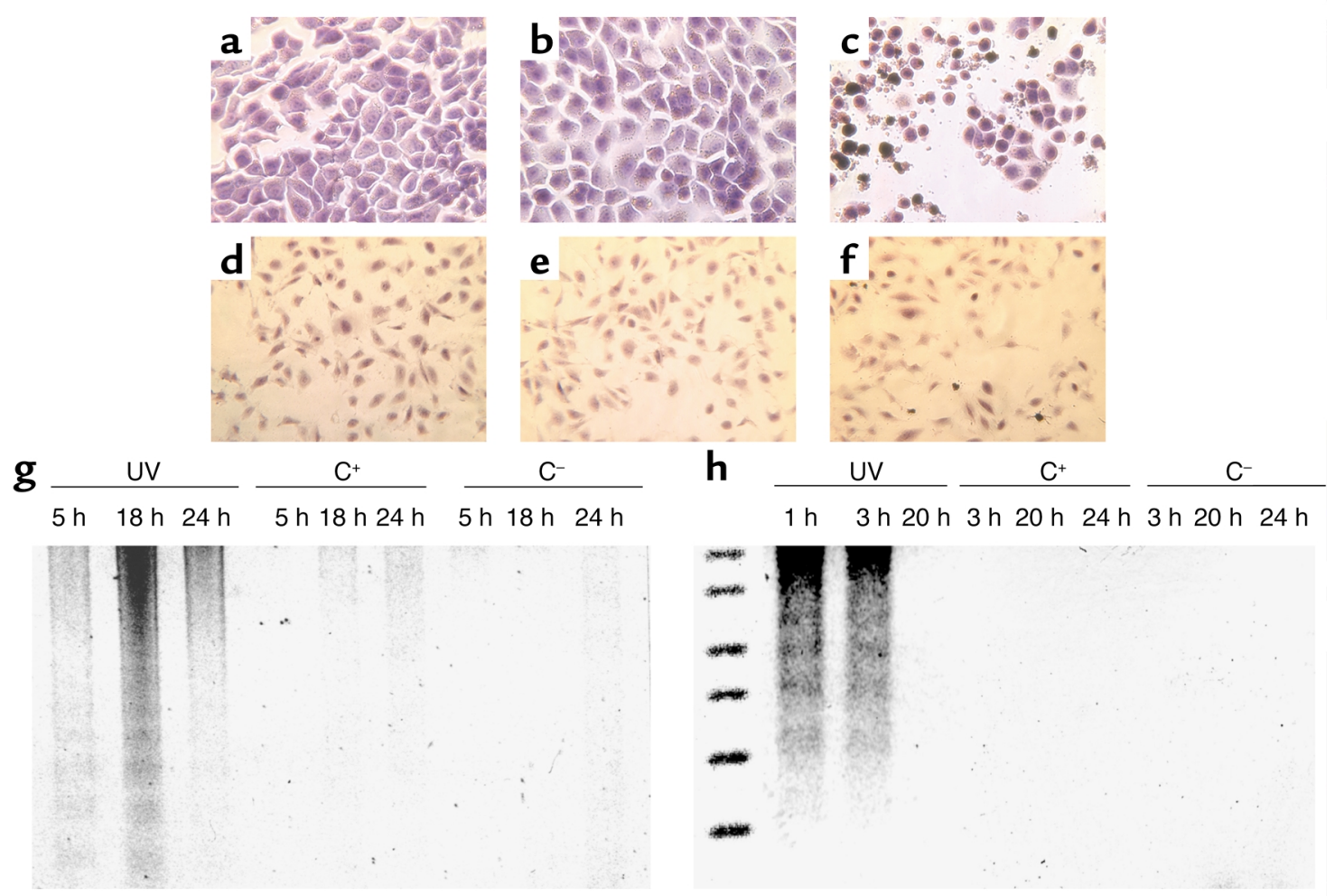

Figure 3

Sublytic C5b-9 does not induce apoptosis in podocytes. a-f show TUNEL staining, a marker of apoptosis. Apoptosis was not detected in control rat (a) and mouse ( $\mathbf{d})$ podocytes exposed to Ab without a complement source, nor in experimental rat (b) and mouse (e) cells exposed to $\mathrm{Ab}$ with a complement source. In contrast, TUNEL staining is increased in rat $(\mathbf{c})$ and mouse ( $\mathbf{f}$ ) podocytes exposed to UV irradiation used as a positive control. The suicide track DNA ladder assay is shown in rat ( $\mathbf{g})$ and mouse $(\mathbf{h})$ podocytes. In contrast to DNA fragmentation in podocytes exposed to UV irradiation (lanes 1, 2, 3), DNA fragmentation was not detected in cells exposed to $A b$ with $\left(C^{+}\right)($lanes $4,5,6)$ and without $\left(\mathrm{C}^{-}\right)$(lanes $\left.7,8,9\right)$ a complement source. 


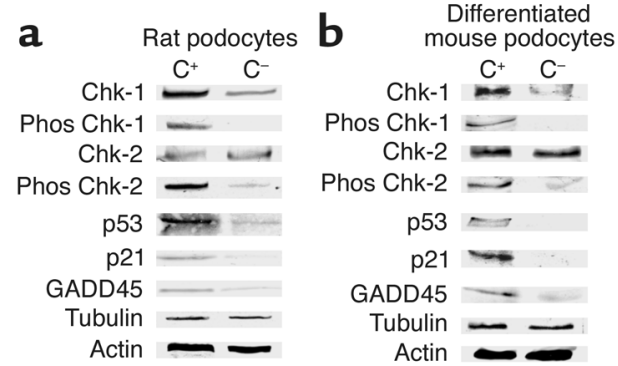

Figure 4

Sublytic C5b-9 increases CHK-1, CHK-2, p53, p21, and GADD45 expression in podocytes in vitro. Western blot analysis was used to measure proteins from in vitro rat (a) and mouse (b) podocytes exposed to $A b$ with a complement source $\left(\mathrm{C}^{+}\right)$and without a complement source $\left(\mathrm{C}^{-}\right)$. C5b-9 increased the protein levels of total CHK-1, phosphorylated (phos) CHK-1, phosphorylated CHK-2, p53, p21, and GADD45. The housekeeping proteins, actin and tubulin, showed that protein loading was equivalent.

Sublytic C5b-9 exposure also causes DNA damage in PHN rats

PHN was induced in Sprague-Dawley, $\mathrm{PVG} \mathrm{C}^{+}$, and PVG $\mathrm{C}^{-}$rats. Immunostaining for sheep IgG was similar in all three strains injected with anti-Fx1A Ab (results not shown). In contrast, C5b-9 staining was not detected in Sprague-Dawley rats injected with normal sheep serum, nor in $\mathrm{PVG} \mathrm{C}^{-}$rats injected with anti-Fx1A Ab (results not shown). As expected, there was a significant increase in proteinuria in Sprague-Dawley rats with PHN (165 \pm $10.4 \mathrm{mg})$ compared with control Sprague-Dawley rats $(14.6 \pm 3.2)(P<0.001$ versus control $)$.

DNA damage in vivo was measured in PHN rats by several methods. There was no detectable glomerular DNA damage in control animals (Figure 2i). In contrast, DNA damage was present in isolated glomeruli from PHN Sprague-Dawley rats (Figure 2j). To prove that DNA damage was a consequence of C5b-9, PHN was also induced in PVG $\mathrm{C}^{+}$and $\mathrm{PVG} \mathrm{C}^{-}$rats. Our data showed that DNA damage was not detected in control PVG $\mathrm{C}^{-}$animals injected with anti-Fx1A Ab (Figure $2 \mathrm{k}$ ). In contrast, Figure 21 shows DNA damage in $\mathrm{PVG} \mathrm{C}^{+}$rats injected with anti-Fx1A Ab. Taken together, the data shows that experimental membranous nephropathy is associated with DNA damage, and this was a consequence of C5b-9 attack.

\section{p53 levels increase in vitro and in vivo} following sublytic C5b-9 attack

Figure 4 shows that p53 protein was detected in very low abundance in rat (Figure 4a) and mouse (Figure $4 b)$ control podocytes in vitro exposed to $\mathrm{Ab}$ without a complement source. In contrast, Figure 4 shows that exposing rat (Figure 4a) and mouse (Figure 4b) podocytes to $\mathrm{Ab}$ and a complement source is associated with a marked increase in $\mathrm{p} 53$ protein levels.

We next determined if C5b-9 attack also increased p53 in vivo, using the PHN model. As shown in Figure 5, p53 immunostaining was not detected in control glomeruli (Figure 5a). In contrast, there was a marked increase in p53 immunostaining in $\mathrm{PHN}$ rats in a podocyte distribution (Figure $5 \mathrm{~b}$ ). Figure 5 also shows a Western blot analysis performed on protein extracted from isolated glomeruli and confirms that p53 protein levels were increased in glomeruli from PHN rats compared with control rats.

To confirm that the increase in $\mathrm{p} 53$ was due to $\mathrm{C} 5 \mathrm{~b}-9$, we also examined p53 expression in $\mathrm{PVG} \mathrm{C}^{-}$and $\mathrm{PVG} \mathrm{C}^{+}$ rats injected with anti-Fx1A Ab. As expected, there was an increase in p53 levels measured by immunostaining and Western blot analysis in PHN PVG $\mathrm{C}^{+}$rats, which was not detected in PVG $\mathrm{C}^{-}$animals injected with antiFx1A (results not shown). Taken together, these results show that p53 expression increases in vitro and in vivo following exposure to $\mathrm{Ab}$ and a complement source and that this effect was a consequence of C5b-9 formation.

Specific cell cycle proteins are increased in podocyte following C5b-9 attack

$p 21^{\text {Cip/Kip }}(p 21)$. Figure 4 shows that the cyclin-dependent kinase (CDK) inhibitor p21 was increased in rat (Figure $4 \mathrm{a}$ ) and mouse (Figure $4 \mathrm{~b}$ ) podocytes exposed to $\mathrm{Ab}$ and a complement source. In contrast, p21 did not increase in control cells exposed to $\mathrm{Ab}$ without a complement source. Immunostaining for $\mathrm{p} 21$ protein levels were also increased in vivo in PHN rats (Figure 5). The increase was confirmed by Western blot analysis performed on protein from isolated glomeruli (Figure 5).
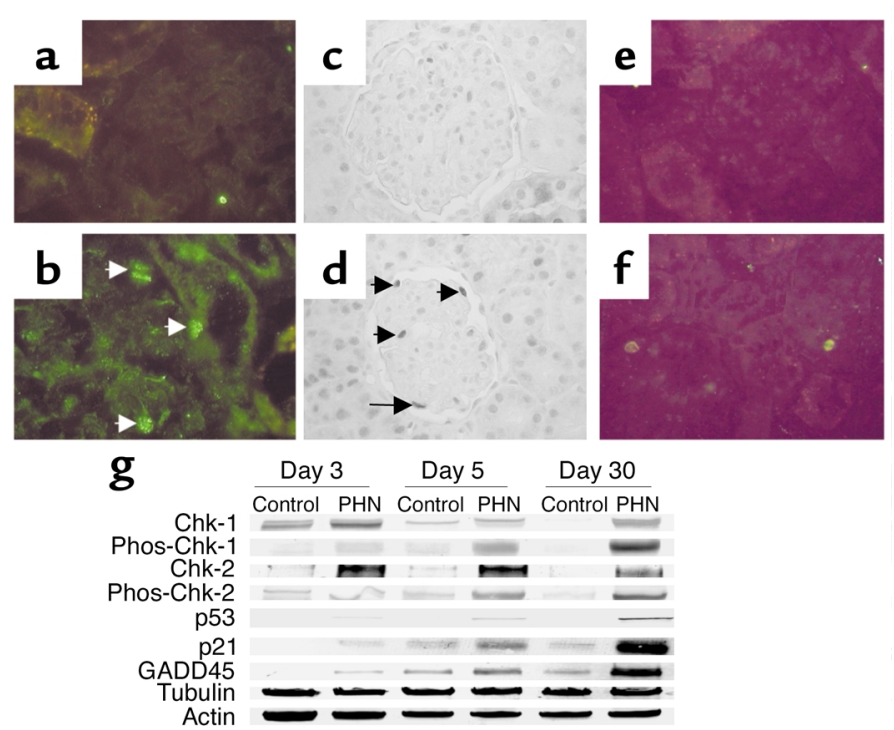

Figure 5

DNA damage proteins increase in PHN rats. Immunostaining for p53 (a), p21 (c), and phosphorylated CHK-2 (e) was not detected in control rats injected with normal sheep serum. In contrast, p53 (b), p21 (d), and phosphorylated CHK-2 immunostaining (f) increased in Sprague-Dawley rats with PHN (arrows indicate positive-stained podocytes). (g) The results of Western blot analysis performed on isolated glomerular protein from control and PHN rats. In PHN animals, the levels for phosphorylated (phos) CHK-1 and CHK-2, p53, p21, and GADD45 increased compared with controls. The housekeeping proteins, actin and tubulin, were used to ensure equal protein loading. 
The levels of $\mathrm{p} 21$ were also increased in $\mathrm{PVG} \mathrm{C}^{+}$rats with PHN measured by immunostaining in a typical podocyte distribution (results not shown). In contrast, p21 expression did not increase in $\mathrm{PVG} \mathrm{C}^{-}$rats injected with anti-Fx1A $\mathrm{Ab}$. These results show that C5b-9 increases $\mathrm{p} 21$ levels in vitro and in vivo.

$G A D D 45$. Figure 4 shows that GADD 45 was detected at low levels in control rat (Figure 4a) and mouse (Figure $4 \mathrm{~b}$ ) podocytes and that levels increased in podocytes following sublytic C5b-9 attack. GADD45 protein was also increased in rats with PHN measured by Western blot analysis (Figure 5) and in $\mathrm{PVG}^{+}$rats with PHN (data not shown). The increase in GADD45 in PHN rats was also detected by immunostaining and the increase localized to podocytes (results not shown). In contrast, GADD45 levels did not increase in PVG $\mathrm{C}^{-}$ rats injected with anti-Fx1A Ab. Taken together, the results show that C5b-9 increases GADD45.

CHK-1 and CHK-2. In rat and mouse podocytes, total CHK-1 and CHK-2 levels were in low abundance in control cells exposed to $\mathrm{Ab}$ without a complement source (Figure 4). In contrast, Figure 4 shows an increase in phosphorylated CHK-1 and CHK-2 in rat (Figure 4a) and mouse (Figure $4 \mathrm{~b}$ ) podocytes in vitro exposed to $\mathrm{Ab}$ and a complement source. The total levels of CHK-1, but not CHK-2, increased following sublytic C5b-9 injury in both rat and mouse podocytes (Figure 4, a and b) Figure 4 also shows that the increase in total and phosphorylated proteins was not due to differences in protein loading, because there was no difference in the protein levels of the housekeeping proteins, tubulin and actin.

The levels of CHK-1 and CHK-2 were also examined in vivo on protein extracted from isolated glomeruli from PHN and control rats. Figure 5 shows that although total CHK-1 and CHK-2 were detected in control and PHN rats, phosphorylated CHK-1 and CHK-2 were not detected in control Sprague-Dawley rats and PVG C- (data not shown). CHK-1 (results not shown) and CHK-2 immunostaining were increased in SpragueDawley rats (Figure 5f) and PVG $\mathrm{C}^{+}$rats (not shown) with PHN, in a typical podocyte distribution. Western blot analysis also showed an increase in phosphorylated CHK-1 and CHK-2 following C5b-9-induced exposure in vivo in PHN Sprague-Dawley rats (Figure 5) and PVG $\mathrm{C}^{+}$rats (data not shown). Results show the phosphorylated (and biologically active) forms of CHK-1 and CHK-2 increase in podocytes in vitro and in vivo following complement attack, and this effect is a consequence of C5b-9 formation.

\section{Inhibiting ERK reduces DNA response genes and increases DNA damage}

Figure 6a shows the temporal expression of phosphorylated ERK-1 and -2, measured by Western blot analysis, following exposure of podocytes in vitro to sublytic C5b-9 attack. We measured ERK immediately (0-45 $\mathrm{min})$ and later (1-20 h) after sublytic injury. There was a biphasic increase in phosphorylated ERK levels within $15 \mathrm{~min}$ and again at $8 \mathrm{~h}$ (Figure $6 \mathrm{~b}$ ). The increase was not due to unequal protein loading, because total ERK-1 and -2 levels were similar in all lanes, as was the housekeeping protein, actin (data not shown).

We next determined the role of ERK in the response to DNA damage induced by C5b-9. Figure 6c shows that inhibiting ERK with U0126 in C5b-9-attacked podocytes reduced the levels of $\mathrm{p} 21$ and GADD45 protein, but not $\mathrm{p} 53$. These results show that ERK regulates the expression of $\mathrm{p} 21$ and GADD45 following sublytic C5b-9-induced injury in podocytes.

Finally, we asked if ERK-1 and -2 was critical in the DNA-damage response to sublytic C5b-9 injury, and the results are shown in Figure 6d. Compared with podocytes undergoing sublytic C5b-9-induced injury, inhibiting ERK-1 and - 2 significantly augmented DNA damage induced by C5b-9. These studies show that ERK-1 and -2 protects podocytes from C $5 b-9$ inducedinjury DNA damage and that this effect is mediated through p21 and GADD45.

\section{Discussion}

Podocytes are the target of immune-mediated injury in glomerular diseases, particularly membranous nephropathy (22). Following sublytic C5b-9 insertion, podocytes increase the levels of growth factors,

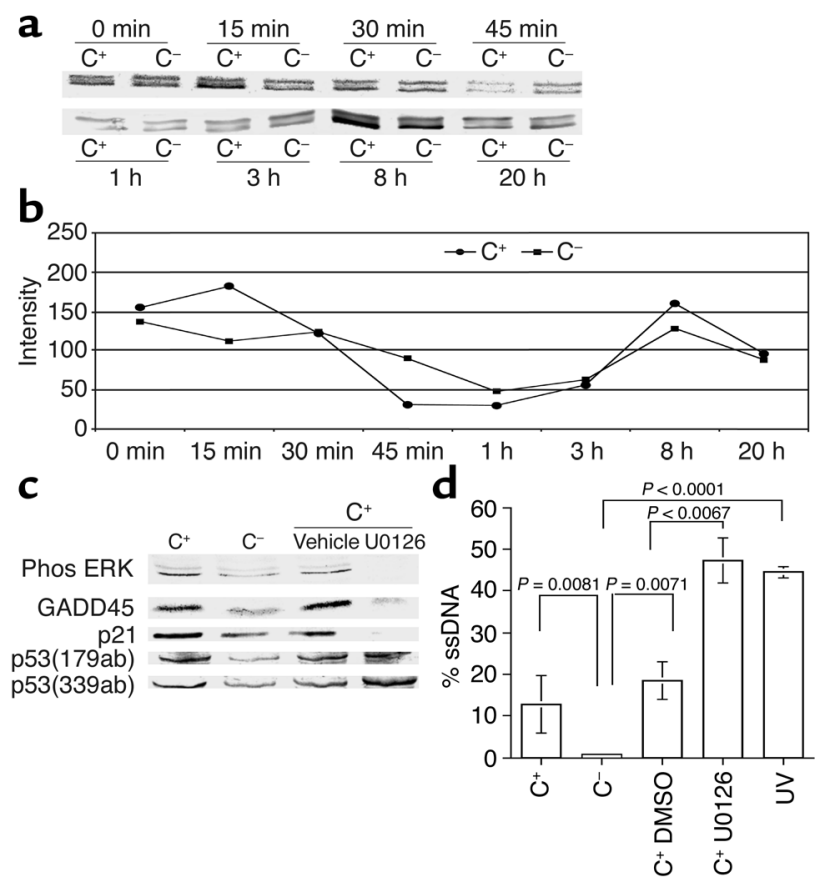

\section{Figure 6}

ERK regulates p 21 and GADD45 following sublytic C5b-9 attack. (a) Phosphorylated ERK was measured by Western blot analysis in control $\left(\mathrm{C}^{-}\right)$and $\mathrm{C} 5 \mathrm{~b}-9$-attacked $\left(\mathrm{C}^{+}\right)$podocytes, and $(\mathbf{b})$ densitometry was used to quantitate the changes compared with total ERK levels. Phosphorylated ERK increased within $15 \mathrm{~min}$ of sublytic C5b-9 attack, and this normalized by $1 \mathrm{~h}$. This was followed by a second increase in phosphorylated ERK at $8 \mathrm{~h}$. (c) Inhibiting phospho-ERK with $U 0126$ reduced GADD45 and p21. (d) Inhibiting ERK-1 and -2 with U0126 augments the DNA damage induced by sublytic C5b-9 (lane 4) compared with controls (lane 3). 
cytokines, and extracellular matrix proteins, activate specific signaling pathways and transcription factors, and release proteases and oxidants (23). Sublytic C5b-9 induces podocyte activation in vitro and in vivo, and this is associated with cell-cycle entry and DNA synthesis (albeit limited) $(6,19)$. In contrast to RMCs $(24)$, however, mitosis and cytokinesis are not marked in podocytes following C5b-9 injury $(7,9,19)$. In this study we report the finding that sublytic C5b-9 causes DNA damage to podocytes in vitro and in vivo, which is associated with an increase in $\mathrm{p} 53$, GADD45, CHK-1, and CHK-2, and CDK-inhibitor p21 and that the MAPK, ERK-1 and -2 protects podocytes from DNA damage induced by C5b-9. The results show that DNA damage is another consequence of cell exposure to sublytic C5b-9 insertion into cell membranes

In contrast to glomerular mesangial and endothelial cells, podocytes do not readily proliferate in response to immune-mediated injury. Moreover, the inadequate proliferative response by podocytes contributes to the development of glomerulosclerosis $(8,25)$. Proliferation is governed at the level of the cell cycle by cell cycle regulatory proteins (26). Podocytes have the nuclear machinery required for DNA synthesis (19). Sublytic C5b-9 attack on podocytes, however, causes a delay/ inhibition in $\mathrm{G}_{2} / \mathrm{M}$ phase (6). Taken together, these studies suggest that the apparent lack of podocyte proliferation occurs both at the level of DNA synthesis and mitosis. Accordingly, the focus of this study was to determine possible mechanisms underlying podocyte $\mathrm{G}_{2} / \mathrm{M}$ phase arrest following C5b-9-induced injury.

The first major finding in the current study was that sublytic C5b-9-induced podocyte injury causes DNA damage in podocytes in vitro and in vivo. The results were similar in rat and differentiated postmitotic mouse podocytes. In contrast, sublytic C5b-9 did not cause DNA damage in RMCs. Moreover, DNA damage was also significantly increased in podocytes in the PHN model of membranous nephropathy, but not in control animals injected with normal sheep serum. Our in vitro and in vivo data show that the phenomenon of DNA damage is C5b-9 dependent. As will be discussed below, our results show that C5b-9 also increases specific DNA damage response genes, further supporting the notion that our findings are specific to DNA damage. In contrast to mesangial cells (27), sublytic C5b-9 injury did not induce apoptosis in cultured rat and mouse podocyte. One explanation is a dose effect, because more lytic concentrations of C5b-9 may have induced apoptosis.

Cells arrest in the cell cycle in order for adequate repair mechanisms to operate following DNA damage. A second major finding in the current study was that sublytic C5b-9-induced injury increased the levels of p53 in mouse and rat podocyte in vitro and in experimental membranous nephropathy in vivo. To date, an increase in p53 levels has only been described in response to injury induced by UV irradiation and certain DNA-damaging agents (28). We can now add sublytic DNA damage to the list of injuries increasing p53.
The CDK inhibitor $\mathrm{p} 21$ inhibits cell cycle progression at $\mathrm{G}_{1} / \mathrm{S}_{\text {and }} \mathrm{G}_{2} / \mathrm{M}(29)$, and $\mathrm{p} 21$ is increased in podocytes in PHN rats (19). Podocyte proliferation is markedly increased in p21-null mice with immune-mediated glomerulonephritis (30). In this study we show that p21 is also increased in podocytes in culture, confirming our findings in vivo in response to sublytic C5b-9. Studies have shown that following DNA damage, p53-dependent increases in p21 are required to halt cell cycle progression to allow adequate time for DNA repair mechanisms (31). Accordingly, we speculate that the increase in $\mathrm{p} 21$ in C5b-9-injured podocytes in vitro and in vivo may be due to increased $\mathrm{p} 53$ expression as a consequence of DNA damage. Increased p21 in turn would inhibit podocyte proliferation at $G_{1} / S$ and $G_{2} / M$ phases.

GADD45 (growth arrest and DNA damage) is a family of genes that has been implicated in the cellular response to DNA damage (32). Our data showed that GADD45 is increased following sublytic injury in vitro and in vivo. Transcription of GADD45, at least partially, is induced by p53 in response to DNA damage induced by UV and ionizing radiation and other DNAdamaging agents. GADD45 binds to proliferating cell nuclear antigen at several residues, thereby causing cell cycle arrest, and GADD45 may also be involved in nuclear excision repair of damaged DNA $(33,34)$. GADD45 is usually expressed at low levels during the S-phase, but is rapidly induced in response to damaged DNA. Protein expression of GADD45 was increased in podocytes exposed in vitro to C5b-9, as well as in glomeruli from PHN rats.

Recent studies have shown that the $G_{2} / M$ phase is also inhibited by a family of proteins known as checkpoint kinase inhibitors (CHK-1 and CHK-2) (35). A third major finding in this study was that sublytic C5b-9-induced podocyte injury increased the levels of CHK- 1 and CHK-2 in vitro and in podocytes in vivo in the PHN model. Of note, CHK-1 levels and activity are regulated in part by p53. These observations have lead us to suggest that the increase in p53 in the current study may also limit podocyte proliferation by increasing $\mathrm{CHK}$ protein levels.

ERK, a member of the MAP kinases, has been shown by Cybulsky et al. to increase in podocytes following sublytic C5b-9 injury (36). The fourth finding in the current study was that inhibiting ERK caused a marked decrease in p21 and GADD45. ERK has been shown to be involved in the signaling response to UV irradiation-induced DNA damage. Studies by Kraus have also shown an increase in phosphorylated ERK in COS and K562 cells in response to sublytic C5b-9 injury (37). Our results, however, showed that inhibiting ERK-1 and -2 augmented the DNA-damage response to sublytic C5b-9 in podocytes. Taken together, our data suggests that following sublytic C5b-9-induced injury, increased ERK activation protects podocytes from DNA damage by increasing p21 and GADD45,

In summary, to examine the possibility that C5b-9induced podocyte injury may cause DNA damage, we 
have used cultured podocytes and the PHN model of membranous nephropathy for our studies, because in this model Ab-complement-induced injury can be examined both in vitro and in vivo in circumstances that mimic the human disease membranous nephropathy. In PHN, IgG Ab to podocyte antigen(s) on the cell membrane binds exclusively to the podocyte and leads to C5b-9 insertion into the podocyte membrane and a consequent nonlytic form of injury that results in proteinuria. The PHN model is C5b-9 dependent and mediated by molecules such as oxidants (38) and proteases $(39,40)$ that are produced by podocytes in response to sublytic C5b-9 attack. The same system of $\mathrm{Ab} / \mathrm{C} 5 \mathrm{~b}-9$ attack can also be replicated with podocytes in vitro. By manipulating the presence or absence of $\mathrm{C} 6$ in these systems, the consequence of C5b-9 attack on podocytes may be examined, and the effect on cell cycle regulatory proteins and DNA damage response genes may be assessed. We conclude that a novel response to sublytic C5b-9 attack on nucleated cells is DNA damage. In the case of podocytes, DNA damage may explain in part the lack of proliferation in certain immunemediated glomerular diseases.

\section{Acknowledgments}

This work was supported by Public Health Service grants (DK34198, DK52121, DK51096, DK56799), a George M. O’Brien Kidney Center grant (DK47659), and a Juvenile Diabetes Research Foundation Award (S.J. Shankland). S.J. Shankland holds an American Heart Association Established Investigator Award. We thank Peter Mundel for the use of mouse podocytes.

1. Couser, W.G. 1990. Mediation of immune glomerular injury. J. Am. Soc. Nephrol. 1:13-29.

2. Cybulsky, A.V., Monge, J.C., Papillon, J., and McTavish, A.J. 1995. Complement C5b-9 activates cytosolic phospholipase A2 in glomerular epithelial cells. Am. J. Physiol. 269:F739-F749.

3. Torbohm, I., et al. 1990. C5b-8 and C5b-9 modulate the collagen release of human glomerular epithelial cells. Kidney Int. 37:1098-1104.

4. Hansch, G.M., Seitz, M., and Betz, M. 1987. Effect of the late complement components C5b-9 on human monocytes: release of prostanoids, oxygen radicals and of a factor inducing cell proliferation. Int. Arch. Allergy Appl. Immunol. 82:317-320.

5. Lovett, D.H., Haensch, G.-M., Goppelt, M., Resch, K.D.G. 1987. Activation of glomerular mesangial cells by the terminal membrane attack complex of complement. J. Immunol. 138:2413-2480.

6. Shankland, S.J., Pippin, J.W., and Couser, W.G. 1999. Complement (C5b-9) induces glomerular epithelial cell DNA synthesis but not proliferation in vitro. Kidney Int. 56:538-548.

7. Floege, J., et al. 1993. Visceral glomerular epithelial cells can proliferate in vivo and synthesize platelet-derived growth factor B-chain. Am.J. Pathol. 142:637-650.

8. Kriz, W. 1996. Progressive renal failure-inability of podocyte to replicate and the consequences for development of glomerulosclerosis. Nephrol. Dial. Transplant. 11:1738-1742.

9. Kriz, W., Hahnel, B., Rosener, S., and Elger, M. 1995. Long-term treatment of rats with FGF-2 results in focal segmental glomerulosclerosis. Kidney Int. 48:1435-1450.

10. Kaufmann, W.K., and Paules, R.S. 1996. DNA damage and cell cycle checkpoints. FASEB J. 10:238-247.

11. Couser, W.G., Pippin, J.W., and Shankland, S.J. 2001. Complement (C5b-9) induces DNA synthesis in rat mesangial cells in vitro. Kidney Int. 59:905-912.

12. Shankland, S.J., et al. 1997. Mesangial cell proliferation mediated by PDGF and bFGF is determined by levels of the cyclin kinase inhibitor p27Kip1. Kidney Int. 51:1088-1099.
13. Mundel, P., Reiser, J., and Kriz, W. 1997. Induction of differentiation in cultured rat and human podocytes. J. Am. Soc. Nephrol. 8:697-705.

14. Brandt, J., et al. 1996. Role of complement membrane attack complex (C5b-9) in mediating experimental mesangioproliferative glomerulonephritis. Kidney Int. 49:335-343.

15. Nangaku, M., et al. 1996. Transfected CD59 protects mesangial cell from injury induced by antibody and complement. Kidney Int. 50:257-266.

16. Shankland, S.J., et al. 1996. Differential expression of transforming growth factor-b isoforms and receptors in experimental membranous nephropathy. Kidney Int. 50:116-124.

17. Leanderson, P., Wennerberg, K., and Tagesson, C. 1994. DNA microfiltration assay: a simple technique for detecting DNA damage in mammalian cells. Carcinogenesis. 15:137-139.

18. Hughes, J., Brown, P., and Shankland, S.J. 1999. The cyclin kinase inhibitor p21CIP1/WAF1 limits interstitial but not tubular cell proliferation in tubulointerstitial injury induced by ureteric obstruction. Am. J. Physiol. 277:F948-F956.

19. Shankland, S.J., et al. 1997. Cyclin kinase inhibitors are increased during experimental membranous nephropathy: potential role in limiting glomerular epithelial cell proliferation in vivo. Kidney Int. 52:404-413.

20. Pippin, J.W., Qu, Q., Meijer, L., and Shankland, S.J. 1997. Direct in vivo inhibition of the nuclear cell cycle cascade in experimental mesangial proliferative glomerulonephritis with roscovitine, a novel cyclindependent kinase antagonist. J. Clin. Invest. 100:2512-2520.

21. Shankland, S.J., et al. 1996. Changes in cell cycle protein expression during experimental mesangial proliferative glomerulonephritis. Kidney Int. 50:1230-1239.

22. Couser, W.G., and Abrass, C.K. 1988. Pathogenesis of membranous nephropathy. Ann. Rev. Med. 39:517-530.

23. Kerjaschki, D. 2001. Caught flat-footed: podocyte damage and the molecular bases of focal glomerulosclerosis. J. Clin. Invest. 108:1583-1587. doi:10.1172/JCI200114629.

24. Floege, J., et al. 1993. Infusion of platelet-derived growth factor or basic fibroblast growth factor induces selective glomerular mesangial cell proliferation and matrix accumulation in rats. J. Clin. Invest. 92:2952-2962

25. Kriz, W., Gretz, N., and Lemley, K.V. 1998. Progression of glomerular diseases: is the podocyte the culprit? Kidney Int. 54:687-697.

26. Roberts, J.M., et al. 1994. Cyclins, CDKs, and cyclin kinase inhibitors. Cold Spring Harbor Symp. Quant. Biol. 59:31-38.

27. Nauta, A.J., et al. 2002. The membrane attack complex of complement induces caspace activation and apoptosis. Eur. J. Immunol. 32:783-792.

28. Hirao, A., et al. 2000. DNA damage-induced activation of $\mathrm{p} 53$ by the checkpoint kinase Chk2. Science. 287:1824-1827.

29. Harper, J.W., et al. 1995. Inhibition of cyclin dependent kinases by $\mathrm{p} 21$. Mol. Biol. Cell. 6:387-400.

30. Kim, Y.-G., et al. 1999. The cyclin kinase inhibitor p21Cip1/WAF1 limits visceral glomerular epithelial cell proliferation in experimental glomerulonephritis. Kidney Int. 55:2349-2361.

31. El-Deiry, W.S., et al. 1993. p21WAF1, a potential mediator of p53 tumor suppression. Cell. 75:817-825.

32. Martin, M.L., et al. 2000. p53-mediated DNA repair responses to UV radiation: studies of mouse cells lacking p53, p 21 and/or gadd 45 genes. Mol. Cell Biol. 20:3705-3714.

33. Smith, M.L., et al. 1994. Interaction of the p53-regulated protein Gadd45 and proliferating cell nuclear antigen. Science. 266:1376-1380.

34. Chen, I.-T., Smith, M.L., O'Connor, P.M., and Fornace, J.A. 1995. Direct interaction of Gadd45 with PCNA and evidence for competitive interaction of Gadd45 and p21(Waf1/Cip1) with PCNA. Oncogene. 11:1931-1937.

35. Liu, Q., et al. 2000. Chk1 is an essential kinase that is regulated by Atr and required for the G2/M DNA damage checkpoint. Genes Dev. 14:1448-1459.

36. Cybulsky, A.V., Papillon, J., and McTavish, A.J. 1998. Complement activates phospholipases and protein kinases in glomerular epithelial cells. Kidney Int. 54:360-372.

37. Kraus, S., Seger, R., and Fishelson, Z. 2001. Involvement of ERK mitogen-activated protein kinase in cell resistance to complement-mediated lysis. Clin. Exp. Immunol. 123:366-374.

38. Neale, T.J., et al. 1994. Proteinuria in passive Heymann nephritis is associated with lipid peroxidation and formation of adducts of type IV collagen. J. Clin. Invest. 94:1577-1584.

39. McMillan, J.I., Riordan, J.W., Couser, W.G., Pollock, A.S., and Lovett, D.H. 1996. Characterization of a glomerular epithelial cell metalloproteinase as matrix metalloproteinase- 9 with enhanced expression in a model of membranous nephropathy. J. Clin. Invest. 97:1094-2101.

40. Johnson, R.J., et al. 1992. Glomerular epithelial cells secrete a glomerular basement membrane-degrading metalloproteinase. J. Am. Soc. Nephrol. 2:1388-1397. 